\title{
Experimental computation with oscillatory integrals
}

\author{
David H. Bailey* Jonathan M. Borwein ${ }^{\dagger}$
}

June 26, 2009

\begin{abstract}
A previous study by one of the present authors, together with D. Borwein and I. Leonard [8], studied the asymptotic behavior of the $p$-norm of the $\operatorname{sinc}$ function: $\operatorname{sinc}(x)=(\sin x) / x$ and along the way looked at closed forms for integer values of $p$. In this study we address these integrals with the tools of experimental mathematics, namely by computing their numerical values to high precision, both as a challenge in itself, and also in an attempt to recognize the numerical values as closed-form constants. With this approach, we are able to reproduce several of the results of [8] and to find new results, both numeric and analytic, that go beyond the previous study.
\end{abstract}

\section{Introduction}

A previous work by one of the present authors, together with D. Borwein and I. Leonard [8], studied the behavior of the $p$-norm of the sinc function: $\operatorname{sinc}(x)=(\sin x) / x$. In particular, these authors considered the function $I(p)$ defined by:

$$
I(p):=\sqrt{p} \int_{0}^{\infty}\left|\frac{\sin t}{t}\right|^{p} \mathrm{~d} t
$$

Plots of $I(p)$ over $(0,10)$ and $(0,100)$ are shown in Figures 1 and 2. In this study we wish to further explore this function, both numerically and analytically. Indeed, in [8] one finds proofs of the following composite result.

Theorem 1 For all $p>1$ one has

$$
I(p)>\sqrt{\frac{3 \pi}{2}} \frac{2 p}{2 p+1}>\sqrt{\frac{3 \pi}{2}}\left(1-\frac{1}{2 p}\right) .
$$

Moreover

$$
\lim _{p \rightarrow \infty} I(p)=\sqrt{\frac{3 \pi}{2}}
$$

and there are real constants $c_{s}$ such that

$$
\begin{aligned}
I(p) & \sim \frac{1}{2} \sqrt{p} \int_{-\pi}^{\pi}\left|\frac{\sin (x)}{x}\right|^{p} d x \\
& \sim \sqrt{\frac{3 \pi}{2}}-\frac{3}{20} \sqrt{\frac{3 \pi}{2}} \frac{1}{p}+\sum_{s=2}^{\infty} c_{s} \frac{1}{p^{s}}+\cdots .
\end{aligned}
$$

${ }^{*}$ Lawrence Berkeley National Laboratory, Berkeley, CA 94720, dhbailey@1bl.gov. Supported in part by the Director, Office of Computational and Technology Research, Division of Mathematical, Information, and Computational Sciences of the U.S. Department of Energy, under contract number DE-AC02-05CH11231.

${ }^{\dagger}$ School of Mathematical and Physical Sciences, University of Newcastle, Callaghan, NSW 2308, Australia jonathan.borwein@newcastle.edu. au and Faculty of Computer Science, Dalhousie University, Halifax, NS, B3H 2W5, Canada, jborwein@cs.dal.ca. Supported in part by ARC, NSERC and the Canada Research Chair Programme. 


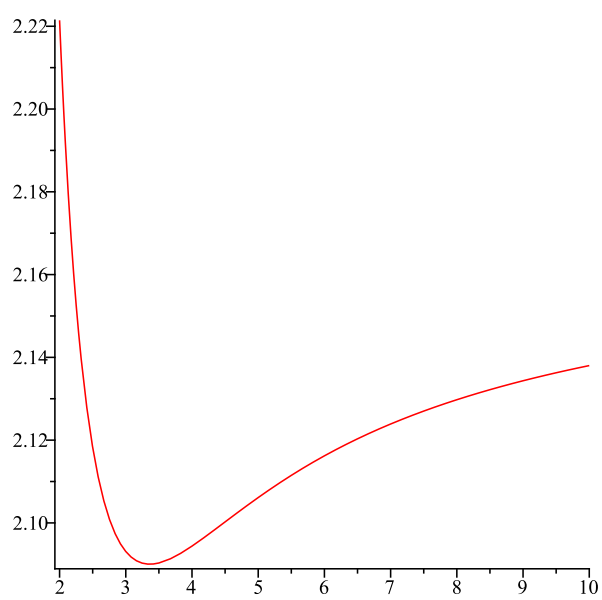

Figure 1: The function $I$ on $[2,10]$

A first challenge we set ourselves was to compute the limit value in (3) sufficiently well to recognize the limit from numerical computation. Given the slow rate of convergence in (4) this is a very hard task, the lower bound being of the correct order. The following related conjecture is made in [8] and is well supported by the plots in Figures 1 and 2 .

Conjecture $I$ is increasing for $p$ above the conjectured global minimum near 3.36 and concave for $p$ above an inflection point near 4.469 .

Note that (4) shows that $I(p)$ is concave increasing for sufficiently large $p$. While we can not fully resolve this conjecture, we are able - inter alia - to resolve both the critical point and inflection point to very high precision. We could not thusly identify a closed form but someone else may be able to determine a closed form for one or both of these quantities.

\section{Quadrature for highly oscillatory integrals}

In a previous study of quadrature algorithms for very high precision, one of the present authors, together with X. S. Li and K. Jeyabalan, found that Gaussian quadrature is generally the most efficient scheme for regular functions (even at endpoints), and for moderately high precision levels (up to several hundred digits). For functions with singularities or other difficulties at the endpoints, or for any function at very high precision (beyond about 500 digits), these authors found that the tanh-sinh quadrature algorithm of Takahasi and Mori is generally the most effective [5] [23].

The tanh-sinh scheme is simply the observation that if $x=g(t)=\tanh (\pi / 2 \sinh t)$, then

$$
\int_{-1}^{1} f(x) d x=\int_{-\infty}^{\infty} f(g(t)) g^{\prime}(t) d t \approx h \sum_{j=-N}^{N} w_{j} f\left(x_{j}\right),
$$

where $x_{j}=g(h j), w_{j}=g(h j)$ and $N$ is chosen large enough that the terms of the summation are smaller than the "epsilon" of the numeric precision being used for $j>N$. Note that the resulting quadrature rule is a simple sum of weighted function values at precalculated abscissas $x_{j}$, with weights $w_{j}$ - very similar to Gaussian quadrature in this regard. Because of the nature of the function $g(t)$, the transformed integrand $f(g(t)) g^{\prime}(t)$ rapidly goes to zero (together with all higher derivatives), even in (most) cases where the original integrand function $f(x)$ has blow-up singularities at the endpoints of the interval $[-1,1]$. Thus tanh-sinh quadrature exhibits "exponential" or "quadratic" convergence for a wide range of integrands - dividing the interval length $h$ by two (or, equivalently, taking twice as many abscissa points) typically doubles the number of correct digits. 


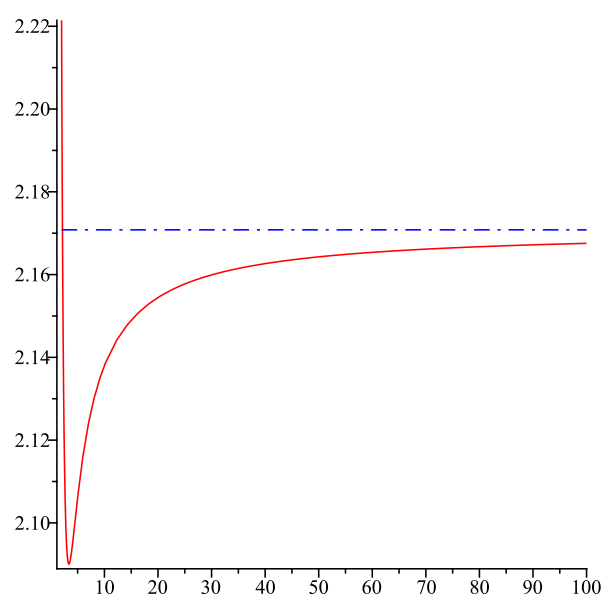

Figure 2: The function $I$ and its limiting value on $[2,100]$

Even armed with advanced quadrature techniques, numerical evaluation of (1) to high precision (say several hundred digits) presents numerous challenges. To begin with, it is first necessary to rewrite this integral as one or more integrals on finite intervals. For most integrands this is handled by a simple transformation such as:

$$
\int_{0}^{\infty} f(t) \mathrm{d} t=\int_{0}^{1} f(t) \mathrm{d} t+\int_{0}^{1} \frac{f(1 / t) \mathrm{d} t}{t^{2}}
$$

Unfortunately, in the case of integrals such as (1), this transformation yields, as the second integrand, the function $\sin (1 / t) / t^{2}$, which oscillates wildly near the origin. Partly for such reasons, this integral (with $p=1$, and without the absolute value) was selected to be one of 15 test integrals in the above-mentioned study of quadrature algorithms [5].

As it turns out, when $p$ is an even integer (so that the absolute value signs can be replaced by simple parentheses), such integrals can be efficiently computed by applying a clever technique recently introduced by Ooura and Mori [20]. Let $x=g(t)=M t /(1-\exp (-2 \pi \sinh t))$. Then in the case of $p=2$, for instance,

$$
\int_{0}^{\infty}\left(\frac{\sin x}{x}\right)^{2} \mathrm{~d} x=\int_{-\infty}^{\infty}\left(\frac{\sin g(t)}{g(t)}\right)^{2} \cdot g^{\prime}(t) \mathrm{d} t \approx h \sum_{k=-N}^{N}\left(\frac{\sin g(h k)}{g(h k)}\right)^{2} \cdot g^{\prime}(h k)
$$

Now note that if one chooses $M=\pi / h$, then for large $k$, the $g(h k)$ values are all very close to $k \pi$, so the $\sin (g(h k))$ values are all very close to zero. Thus the sum can be truncated after a modest number of terms, as in tanh-sinh quadrature. In practice, this scheme is very effective for oscillatory integrands such as this.

When $p$ is not an even integer, neither than tanh-sinh nor the Ooura-Mori scheme can be used, because the condition of regularity except at endpoints is not met due to the absolute value signs in (1). What happens is that convergence is very slow, and even with high precision arithmetic and many abscissa-weight pairs, accuracy is typically only a few digits. One response is to write, for integer $N$ and $2 N-1>|\theta|$,

$$
\int_{0}^{\infty}\left(\left|\frac{\sin (t)}{t}\right|\right)^{2 N+\theta} \mathrm{d} t=\sum_{n=0}^{\infty} \frac{\theta^{n}}{n ! 2^{n}} \int_{0}^{\infty}\left(\frac{\sin (t)}{t}\right)^{2 N}\left(\log \left(\frac{\sin (t)}{t}\right)^{2}\right)^{n} \mathrm{~d} t
$$

on expanding the $\theta$-power as an exponential series. This eliminates the absolute value signs, but unfortunately the $\log$ sin term results in singularities at every integer multiple of $\pi$, and thus, sadly, is also not 
suitable for high-precision quadrature computation as it stands. We shall return to this type of integral in Section 4.

An alternate strategy is to employ the Hurwitz zeta function, which is defined as:

$$
\zeta(p, x) \quad:=\sum_{n=0}^{\infty} \frac{1}{(n+x)^{p}}
$$

With this definition, we can derive:

$$
I(p):=\sqrt{p} \int_{0}^{\infty}\left|\frac{\sin (x)}{x}\right|^{p} d x=\frac{\sqrt{p}}{\pi^{p-1}} \int_{0}^{1} \sin ^{p}(\pi x) \zeta(p, x) d x .
$$

This can be obtained by breaking the integral in (8) on the intervals $[n \pi,(n+1) \pi]$ and gathering up the translations.

We recall that the Bateman project $[15,(7)$, p. 24] records

$$
\zeta(s, v)=\frac{1}{\Gamma(s)} \int_{0}^{1} \frac{x^{v-1}(-\ln (x))^{s-1}}{1-x} d x .
$$

An even more efficient scheme is given by the following formula supplied by Richard Crandall [3]:

Lemma 1 (Crandall) The complete analytic continuation of $\zeta(s, a)$ for $a \in(0,1), s \neq 1+0 i$, is given by

$$
\zeta(s, a)=\frac{1}{\Gamma(s)} \sum_{n \geq 0} \frac{\Gamma(s, \lambda(n+a))}{(n+a)^{s}}+\frac{1}{\Gamma(s)} \sum_{m \geq 0} \frac{(-1)^{m} B_{m}(a)}{m !} \frac{\lambda^{m+s-1}}{m+s-1},
$$

with the following interpretations: $\Gamma(s, \cdot)$ is the standard incomplete gamma function, $B_{n}$ is the standard Bernoulli polynomial, $\lambda$ is a free parameter with $|\lambda|<2 \pi$. For any case of integer $s=-n \leq 0$, the $\Gamma(s)$ divergence cancels a divergent $m$-summand, and so $\zeta(-n, a)=-B_{n+1}(a) /(n+1)$.

We chose not to use this formula because it requires access to the incomplete gamma function. Instead, for our purposes here it suffices to use the Euler-Maclaurin summation formula [2, pg. 180]. Let $m \geq 0$ and $n \geq 1$ be integers, and define $h=(b-a) / n$ and $x_{j}=a+j h$ for $0 \leq j \leq n$. Further assume that the function $f(x)$ is at least $(2 m+2)$-times continuously differentiable on $[a, b]$. Then the Euler-Maclaurin summation formula is:

$$
h \sum_{j=0}^{n} f\left(x_{j}\right)=\int_{a}^{b} f(x)+\frac{h}{2}(f(a)+f(b))+\sum_{i=1}^{m} \frac{h^{2 i} B_{2 i}}{(2 i) !}\left(f^{(2 i-1)}(b)-f^{(2 i-1)}(a)\right)+E
$$

where $B_{2 i}$ denote the Bernoulli numbers, and

$$
E=\frac{h^{2 m+2}(b-a) B_{2 m+2} f^{2 m+2}(\xi)}{(2 m+2) !}
$$

for some $\xi \in(a, b)$.

As written, this formula is not particularly effective to numerically evaluate series. The strategy is instead to evaluate a series manually for, say, $N=1000$ terms, then to use the Euler-Maclaurin formula to evaluate only the tail of the series, namely $\sum_{j>N} f\left(x_{j}\right)$. The Bernoulli numbers $B_{2 k}$, which are required here, can be easily computed by recalling that for $k>0$ [1, pg. 807],

$$
\zeta(2 k)=\frac{(2 \pi)^{2 k}\left|B_{2 k}\right|}{2(2 k) !} .
$$

This can be rewritten as

$$
\frac{B_{2 k}}{(2 k) !}=\frac{2(-1)^{k+1} \zeta(2 k)}{(2 \pi)^{2 k}} .
$$




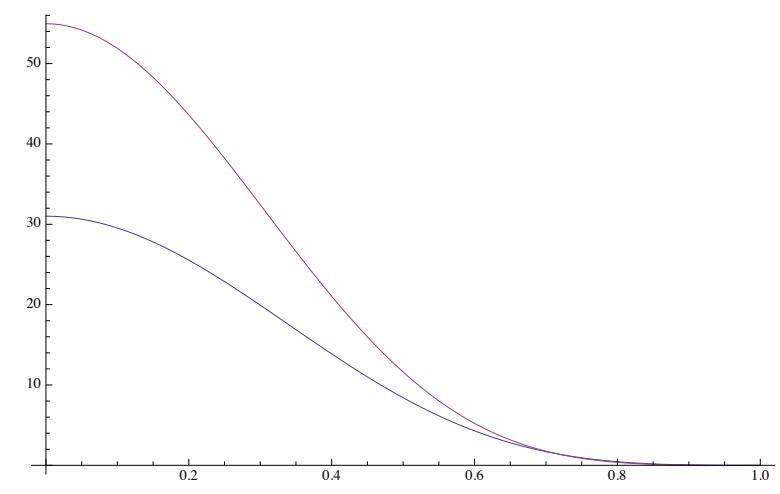

Figure 3: Integrand function for $p=3$ (blue) and $p=3.5$ (red).

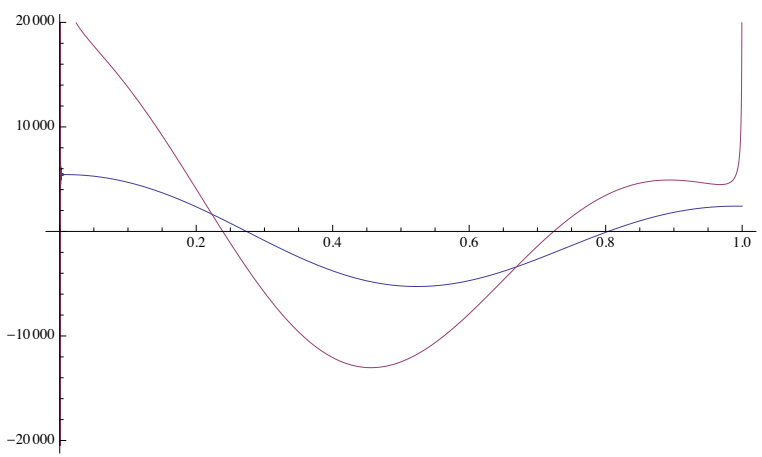

Figure 4: Fourth derivative of integrand function for $p=3$ (blue) and $p=3.5$ (red).

The Riemann zeta function at integer arguments can, in turn, be computed using the formula due to Peter Borwein [10].

With a scheme to compute the Hurwitz zeta in hand, formula (10) can be used, in conjunction with a high-precision quadrature routine, to compute $I(p)$.

One question here is whether to use Gaussian quadrature or tanh-sinh. Because the integrand function $\sin ^{p}(\pi x) \zeta(p, x)$ for various $p$ seems quite well-behaved, our first inclination was to use Gaussian quadrature. This works quite well for $p=3$, for instance, but then we found that it gave very poor results for $p=3.5$ - even with a very small $h$ and a correspondingly large number of evaluation points, the accuracy was only a few digits. We naturally suspected a programming "bug," but after observing the same behavior whether we coded the algorithm in our own ARPREC software [4] or in Mathematica, we looked elsewhere.

As it turns out, this strange behavior is due to the fact that when $p=3.5$, while the integrand function and its derivatives are well-behaved up to the third derivative, the fourth derivative exhibits severe blowup singularities at 0 and 1 . This is illustrated in Figure 3, which shows the function $\sin ^{p}(\pi x) \zeta(p, x)$ for $p=3$ (blue) and $p=3.5$ (red), and Figure 4, which shows the fourth derivatives of these functions with the same color scheme. Because of these singularities in the higher-order derivatives, Gaussian quadrature gives poor results. The tanh-sinh scheme, in contrast, is not bothered by these singularities and gives excellent exponential convergence to high-precision values.

Our first goal with this computer program was to find the minimum of the function $I(p)$ near $p=3.36$ and also the point of inflection near $p=4.46$. We did this by starting with a "comb" of four equispaced abscissa values (five in the case of the inflection point) near 3.36 (4.46 for the inflection point). We evaluated $I(p)$ for these values of $p$, then used polynomial regression to fit these $(p, I(p))$ pairs to a quartic curve (quintic for the inflection point). We then used Newton iteration to calculate a new 
estimate for the zero of the derivative of this function (zero of the second derivative, for the inflection point). This new estimate for the $p$ at the critical point was then taken as a new element of the "comb," and the process was repeated, until successive new elements differed by an amount roughly equal to the square root of the "epsilon" of the numeric precision being used, which is as good as can be expected.

This scheme appeared to work quite well. We obtained the results below and believe them to be accurate to the precision shown:

- $p$ at critical point (conjectured minimum):

3.36354876022451532816334301553541106982340973010200

93393024274526853624322808822111780630522743546839 65168546672961485462827077846841786411218613089950 8745727158152731

- $I(p)$ at critical point (conjectured minimum): 2.09002860269180412254956491550781177353834974949186 75161558946115770419271274624491776411344314758189 93461306711846030747363223735023118868888017902470 29802232734781888386061734850631082243846394257215 38511911622108100945818827513170410889481080593453 364388301851618971531246883340068963419076

- $p$ at inflection point:

4.46987788658564578917780820674988693171596919867299 11634253975525983837941459705451646979509928424279 4233718363336416486397093

\section{The sinc norm at integer values}

For integer $M \geq 1$, on expressing $\zeta(M, x)$ in terms of $\Psi^{(M-1)}(x)$ and integrating by parts $M$-times we have also the representation

$$
I(M)=\frac{\sqrt{M}}{\pi^{M-1}} \int_{0}^{1} \sin ^{M}(\pi x) \zeta(M, x) d x=\frac{\sqrt{M}}{\Gamma(M) \pi^{M-1}} \int_{0}^{1} \log \Gamma(x) \frac{\partial^{M}}{\partial x^{M}} \sin ^{M}(\pi x) d x .
$$

Note also that when $M$ is even we may substitute $x=1-x$ and average to get

$$
I(2 N)=\frac{\sqrt{2 N}}{\pi^{2 N-1}} \int_{0}^{1} \sin ^{2 N}(\pi x) \zeta(2 N, x) d x=-\frac{\sqrt{N / 2}}{\Gamma(2 N) \pi^{2 N-1}} \int_{0}^{1} \log \left(\frac{\sin (\pi x)}{\pi}\right) \frac{\partial^{2 N}}{\partial x^{2 N}} \sin ^{2 N}(\pi x) d x .
$$

We can to use these last two formulae - via their Fourier expansions and Parseval's formula - to recapture the known closed form for even integers and to provide something new for odd integers. Indeed we have

$$
\begin{aligned}
-\ln \left(\frac{\sin (\pi t)}{\pi}\right) & =\log (2 \pi)+\sum_{n=1}^{\infty} \frac{\cos (2 n \pi t)}{n} \\
\frac{1}{2}-t & =\frac{1}{\pi} \sum_{n=1}^{\infty} \frac{\sin (2 n \pi t)}{n} \\
\log \Gamma(t) & =-\frac{1}{2} \log \left(\frac{\sin (\pi t)}{\pi}\right)+(\gamma+\ln (2 \pi))\left(\frac{1}{2}-t\right)+\frac{1}{\pi} \sum_{n=1}^{\infty} \frac{\log (n)}{n} \sin (2 n \pi t)
\end{aligned}
$$

where the final composite Fourier series is originally due to Kummer [13]. Also, it is known [7] and easy to show inductively that

$$
\sin ^{2 N-1}(\pi t)=\frac{1}{2^{2 N-2}} \sum_{n=1}^{N}(-1)^{n+1}\left(\begin{array}{c}
2 N-1 \\
N-n
\end{array}\right) \sin ((2 n-1) \pi t)
$$

and

$$
\sin ^{2 N}(\pi t)=\frac{1}{2^{2 N-1}}\left(\frac{1}{2}\left(\begin{array}{c}
2 N \\
N
\end{array}\right)+\sum_{n=1}^{N}(-1)^{n}\left(\begin{array}{c}
2 N \\
N-n
\end{array}\right) \cos (2 n \pi t)\right)
$$


Finally, for completeness, we recall that for $0 \leq t \leq 1$,

$$
\sum_{n=1}^{\infty} \frac{\sin (2 \pi n t)}{n^{2 N+1}}=\frac{(-1)^{N-1}}{2}(2 \pi)^{2 N+1} \phi_{2 N+1}(t)
$$

and

$$
\sum_{n=1}^{\infty} \frac{\cos (2 \pi n t)}{n^{2 N}}=\frac{(-1)^{N-1}}{2}(2 \pi)^{2 N} \phi_{2 N}(t),
$$

where $\phi_{N}(x)$ is the $N$ th Bernoulli polynomial, normalized so that the highest-order coefficient is $1 / N$ !, see [22, p. 430]. Now it transpires that the first two terms of Kummer's formula (16) and (17) are orthogonal to (19) and hence

$$
\begin{aligned}
I(2 N-1) & =\frac{\sqrt{2 N-1}}{\Gamma(2 N-1)(2 \pi)^{2 N-2}} \sum_{n=1}^{\infty} \int_{0}^{1} \frac{\log (n)}{n} \sin (2 n \pi t) \frac{\partial^{2 N-1}}{\partial x^{2 N-1}} \sin ^{2 N-1}(\pi x) d x \\
& =\frac{1}{\pi} \frac{\sqrt{2 N-1}}{\Gamma(2 N-1)(2 \pi)^{2 N-2}} \sum_{n=1}^{\infty} \sum_{m=1}^{N} \frac{(-1)^{m+1}\left(\begin{array}{c}
2 N-1 \\
N-m
\end{array}\right) \log (n)}{n} \\
& \times \int_{0}^{1} \sin (2 n \pi t) \frac{\partial^{2 N-1}}{\partial x^{2 N-1}} \sin ((2 m-1) \pi t) d x \\
& =\frac{(-1)^{N} \sqrt{2 N-1}}{\Gamma(2 N-1) 4^{N-1}} \sum_{n=1}^{\infty} \sum_{m=1}^{N} \frac{(-1)^{m}\left(\begin{array}{l}
2 N-1 \\
N-m
\end{array}\right)(2 m-1)^{2 N-1} \log (n)}{n} \\
& \times \int_{0}^{1} \sin (2 n \pi t) \cos ((2 m-1) \pi t) d x \\
& =\frac{1}{\pi} \frac{(-1)^{N} \sqrt{2 N-1}}{\Gamma(2 N-1)} \sum_{n=1}^{\infty} \sum_{m=1}^{N}(-1)^{m}\left(\begin{array}{l}
2 N-1 \\
N-m
\end{array}\right)\left(m-\frac{1}{2}\right)^{2 N-1} \frac{\log (n)}{n^{2}-\left(m-\frac{1}{2}\right)^{2}} \\
& =\frac{1}{\pi} \frac{(-1)^{N} \sqrt{2 N-1}}{\Gamma(2 N-1)} \sum_{m=1}^{N}(-1)^{m}\left(\begin{array}{l}
2 N-1 \\
N-m
\end{array}\right)\left(m-\frac{1}{2}\right)^{2 N-1} \sum_{n=1}^{\infty} \frac{\log (n)}{n^{2}-\left(m-\frac{1}{2}\right)^{2}} .
\end{aligned}
$$

Let us set

$$
\mathcal{L}(x):=\sum_{n=2}^{\infty} \frac{\log (n)}{n^{2}-x^{2}}=-\sum_{s=1}^{\infty} \zeta^{\prime}(2 s) x^{2 s-2},
$$

where the power series has radius of convergence two, and the derivative (here and elsewhere) is with respect to the first variable. For general $x$, we may similarly write

$$
\mathcal{L}(x)=\sum_{n=2}^{\lfloor x\rfloor} \frac{\log (n)}{n^{2}-x^{2}}-\sum_{s=1}^{\infty} \zeta^{\prime}(2 s,\lceil x\rceil) x^{2 s-2},
$$

with radius of convergence $\lceil x\rceil$. Thence we obtain

$$
I(2 N-1)=\sqrt{2 N-1} \cdot \frac{(-1)^{N}}{(2 N-2) !} \cdot \sum_{m=1}^{N}(-1)^{m}\left(\begin{array}{c}
2 N-1 \\
N-m
\end{array}\right)\left(m-\frac{1}{2}\right)^{2 N-1} \frac{\mathcal{L}\left(m-\frac{1}{2}\right)}{\pi} .
$$

In particular $I(1)=\infty$ and

$$
\begin{aligned}
I(3) & =\frac{3 \sqrt{3}}{2 \pi} \sum_{s=1}^{\infty} \frac{9^{s}-1}{4^{s}} \zeta^{\prime}(2 s) \\
& =2.0930867689497938424321336535746596878058055573140 \ldots \\
I(5) & =-\frac{5 \sqrt{5}}{64 \pi}\left(\frac{1}{3} \mathcal{L}\left(\frac{1}{2}\right)-\frac{81}{2} \mathcal{L}\left(\frac{3}{2}\right)+\frac{625}{6} \mathcal{L}\left(\frac{5}{2}\right)\right) \\
& =2.1061252846080842088918826986669843292200478501493 \ldots,
\end{aligned}
$$


since

$$
-\mathcal{L}\left(\frac{5}{2}\right)=\frac{4}{9} \log (2)+\sum_{s=1}^{\infty}\left(\zeta^{\prime}(2 s)+\frac{\log (2)}{2^{2 s}}\right)\left(\frac{5}{2}\right)^{2 s-2}
$$

while $\mathcal{L}\left(\frac{1}{2}\right)$ and $\mathcal{L}\left(\frac{3}{2}\right)$ are as already engaged in $(27)$. Such computations can be done quite efficiently to much higher precision in either Maple or Mathematica. Richard Crandall has noted the following pretty formula

$$
I(3)=\frac{3 \sqrt{3}}{8} \pi+6 \sqrt{3} \int_{0}^{\infty} \frac{t^{2}}{\left(t^{2}+1\right)\left(t^{2}+9\right)\left(\mathrm{e}^{\pi t}-1\right)} d t .
$$

Herein, the integral term only accounts for $2.5 \%$ of the value.

For even integers the computation is much simpler as the Fourier series have only cosine terms and so are orthogonal on $[0,1]$. We arrive at:

$$
I(2 N)=\sqrt{2 N} \int_{0}^{\infty}\left(\frac{\sin x}{x}\right)^{2 N} d x=\sqrt{2 N} \cdot \frac{(-1)^{N}}{(2 N-1) !} \cdot \sum_{m=0}^{N}(-1)^{m}\left(\begin{array}{c}
2 N \\
N-m
\end{array}\right) m^{2 N-1} \frac{\pi}{2} .
$$

Hence $I(2)=\pi / 2, I(4)=2 \pi / 3=2.0943951023932 \ldots$ and $I(6)=11 \sqrt{6} \pi / 40=2.1162072197671 \ldots$ Result (30) can be found in Bromwich [11, Exercise 22, p. 518], where it is attributed to Wolstenholme, and in many other places - including two relatively recent articles on integrals of more general products of sinc functions $[6,9]$. We recapitulate with a theorem.

Theorem 2 For integer $N \geq 1$ one has

$$
I(2 N-1)=\sqrt{2 N-1} \cdot \frac{(-1)^{N}}{(2 N-2) !} \cdot \sum_{m=1}^{N}(-1)^{m}\left(\begin{array}{c}
2 N-1 \\
N-m
\end{array}\right)\left(m-\frac{1}{2}\right)^{2 N-1} \frac{\mathcal{L}\left(m-\frac{1}{2}\right)}{\pi},
$$

with $\mathcal{L}$ given by (25), while

$$
I(2 N)=\sqrt{2 N} \int_{0}^{\infty}\left(\frac{\sin x}{x}\right)^{2 N} d x=\sqrt{2 N} \cdot \frac{(-1)^{N}}{(2 N-1) !} \cdot \sum_{m=0}^{N}(-1)^{m}\left(\begin{array}{c}
2 N \\
N-m
\end{array}\right) m^{2 N-1} \frac{\pi}{2} .
$$

The limit revisited For the limit of the $I(p)$, as $p \rightarrow \infty$ the best we could obtain is $\mathbf{2 . 1 7 0 8 0 3 7 5 3 7 \ldots .}$ This required computing $I(p)$ to high precision, using the Hurwitz integral representation (10), for $p=4,16, \ldots$, up to $p=4^{12}$, and then using Richardson extrapolation. This is consistent with the actual

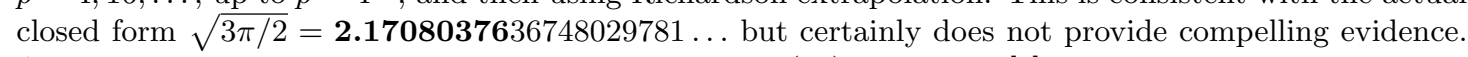
A discussion of the problems entailed in using formula (32) is given in [7].

\section{More general oscillatory integrals}

More complex integrands of the form studied in [6] can be handled by variations of (10). For example

$$
\begin{aligned}
\int_{-\pi}^{\infty}\left|\frac{\sin (x)}{x}\right|^{p}\left|\frac{\sin (x / 2)}{x / 2}\right|^{q} d x & =\frac{2}{(2 \pi)^{p+q-1}} \int_{-1}^{1} \sin ^{p}(\pi x) \sin ^{q}\left(\pi \frac{x}{2}\right) \zeta\left(p+q, \frac{x}{2}\right) d x \\
& +\int_{0}^{\pi}\left(\frac{\sin (x)}{x}\right)^{p}\left(\frac{\sin (x / 2)}{x / 2}\right)^{q} d x .
\end{aligned}
$$

Likewise, logarithmic integrals of the form in (8) can be treated as follows. For $p>1, q \geq 0$ and $q$ 
integer we write

$$
\begin{aligned}
I(p, q) & :=\int_{0}^{\infty}\left|\frac{\sin (t)}{t}\right|^{p}\left(\log \left|\frac{\sin (t)}{t}\right|\right)^{q} d t \\
& =\frac{1}{\pi^{p-1}} \int_{0}^{\infty}\left|\frac{\sin (\pi t)}{t}\right|^{p}\left(\log \left|\frac{\sin (\pi t)}{\pi}\right|-\log |t|\right)^{q} d t \\
& =\frac{1}{\pi^{p-1}} \sum_{r=0}^{q}\left(\begin{array}{c}
q \\
r
\end{array}\right)(-1)^{r} \int_{0}^{\infty}\left|\frac{\sin (\pi t)}{t}\right|^{p}\left(\log \left|\frac{\sin (\pi t)}{\pi}\right|\right)^{q-r}(\log t)^{r} d t \\
& =\frac{1}{\pi^{p-1}} \sum_{r=0}^{q}\left(\begin{array}{c}
q \\
r
\end{array}\right)(-1)^{r} \int_{0}^{\infty}|\sin (\pi t)|^{p}\left(\log \left|\frac{\sin (\pi t)}{\pi}\right|\right)^{q-r} \frac{(\log t)^{r}}{t^{p}} d t \\
& =\frac{1}{\pi^{p-1}} \sum_{r=0}^{q}\left(\begin{array}{c}
q \\
r
\end{array}\right)(-1)^{r} \sum_{n=0}^{\infty} \int_{0}^{1} \sin ^{p}(\pi t) \log ^{q-r}\left(\frac{\sin (\pi t)}{\pi}\right) \frac{\log ^{r}(n+t)}{(n+t)^{p}} d t
\end{aligned}
$$

where this last expression has again broken up the integral. On exchanging the integral and sum, we we arrive at our final theorem:

Theorem 3 For all real numbers $p>1$ and integers $q \geq 0$ one has

$$
\int_{0}^{\infty}\left|\frac{\sin (t)}{t}\right|^{p} \log ^{q}\left|\frac{\sin (t)}{t}\right| d t=\frac{1}{\pi^{p-1}} \sum_{r=0}^{q}\left(\begin{array}{l}
q \\
r
\end{array}\right) \int_{0}^{1} \sin ^{p}(\pi t) \log ^{q-r}\left(\frac{\sin (\pi t)}{\pi}\right) \zeta^{(r)}(p, t) d t
$$

where derivatives of the Hurwitz zeta function are taken with respect to the first variable.

Observe that for computational purposes it is preferable to write

$$
\int_{0}^{\infty}\left|\frac{\sin (t)}{t}\right|^{p} \log ^{q}\left|\frac{\sin (t)}{t}\right| d t=\frac{1}{\pi^{p-1}} \int_{0}^{1} \sin ^{p}(\pi t) \sum_{r=0}^{q}\left(\begin{array}{l}
q \\
r
\end{array}\right) \log ^{q-r}\left(\frac{\sin (\pi t)}{\pi}\right) \zeta^{(r)}(p, t) d t .
$$

Note that formula (36) recaptures (10). Observe also that the Fourier series of $\log \left(\frac{\sin (\pi t)}{\pi}\right)$ was recorded in equation (16). To illustrate the virtues of Theorem 3 , with $p=3$ and $q=1,2,3$ we computed in Maple that

$$
\begin{aligned}
& I(3,1)=\quad-\quad 0.21269444360025161284678764161199575472915948205259008888 \\
& 3795304046951433904376033124895536374597320 \\
& I(3,2)=\quad+\quad 0.15046077234624478828956577555259908739964416463107895870 \\
& 0949532207087021383282901213520074598205646 \\
& I(3,3)=\quad-\quad 0.21673166110791925450854193518248175083146189217945509989 \\
& 946163130368897719634815944215473455858421
\end{aligned}
$$

Each hundred digit computation took roughly three minutes on a modern laptop.

We can also handle quite general sinc products of the sort analyzed in [6]. Let $0<n_{1} \leq n_{2} \leq \cdots \leq n_{N}$ be natural numbers. Set $L:=\operatorname{LCM}\left(n_{1}, n_{2}, \ldots, n_{N}\right)$ and set $M:=n_{1} \cdot n_{2} \cdot \ldots \cdot n_{N}$. Then

$$
\int_{0}^{\infty} \prod_{k=1}^{N} \operatorname{sinc}\left(\frac{x}{n_{k}}\right) d x=\frac{M / L}{(2 L \pi)^{N-1}} \int_{0}^{1} \prod_{k=1}^{N} \sin \left(\frac{2 L}{n_{k}} \pi x\right) \zeta(N, x) d x .
$$

For example

$$
\frac{1}{51840000 \pi^{6}} \int_{0}^{1} \sin ^{4}(10 \pi x) \sin ^{3}(6 \pi x) \Psi(6, x) d x=-2.81369873784840103 \ldots=-\frac{286601}{320000} \pi .
$$

Equation (37) is most efficacious when the number of distinct integers $n_{k}$, and hence $L$, is small. The factor of 2 is not needed when each integer occurs an even number of times. To conclude, we illustrate in Figure 5 the effect of the Hurwitz kernel for $\sin ^{2}(x) / x^{2}$. The increases in the values of the function on $[0,1]$ exactly balance the amount needed to compensate for the values on $[1, \infty]$. 


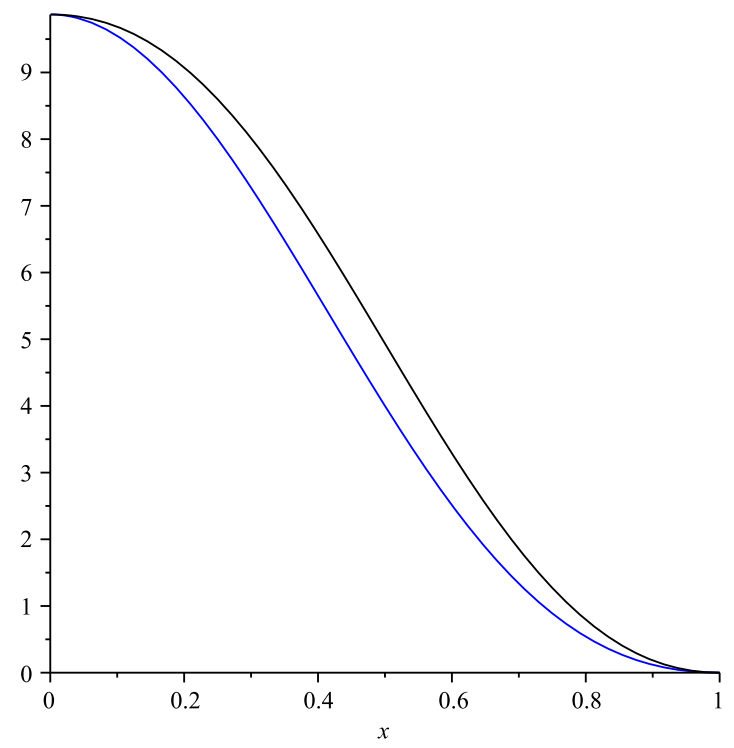

Figure 5: $\frac{\sin ^{2}(\pi x)}{x^{2}}$ (blue) and $\sin ^{2}(\pi x) \zeta(2, x)$ (black).

\section{Concluding remarks}

We found this study stimulating for several reasons.

1. Such numerical computations to high or extreme precision are quite difficult and worthwhile challenges in themselves.

2. In the integer cases for which we have a closed form we obtain excellent test integrals for different high-precision numerical methods.

3. The numerical tools presented new analytic opportunities such as the discovery of the formula (26) which represented an unexpected windfall. It is probably possible to emulate that result for integer $p, q$ in (33).

4. One other unexpected discovery in this study is the fact that the function $\sin ^{p}(\pi t) \zeta(p, t)$ (when $p=3.5)$ is a striking example of a function that appears entirely smooth and well-behaved, but which in fact cannot be integrated using Gaussian quadrature to more than a few digits, due to bad behavior of a higher derivative (the fourth derivative in this case). And for the same reason it is yet one more example of the power of the tanh-sinh quadrature algorithm, since the tanh-sinh scheme integrates this function without difficulty to very high precision.

5. Even partial failures, like the limited success in computing $\lim _{p \rightarrow \infty} I(p)$, have left us with a much better understanding of the issues involved with such experimental techniques.

6. Finally, our inability to determine closed forms for the critical point or the inflection point has still resulted in high-precision data sets that can be revisited if and when inspiration strikes. 


\section{References}

[1] M. Abramowitz and I.A. Stegun, Handbook of Mathematical Functions, NBS (now NIST), 1965. See also http://dlmf.nist.gov.

[2] Kendall E. Atkinson, Elementary Numerical Analysis, John Wiley and Sons, 1993.

[3] David H. Bailey, Jonathan M. Borwein and Richard E. Crandall, "Resolution of the Quinn-Rand-Strogatz Constant of Nonlinear Physics," Experimental Mathematics, to appear, Jun 2007, available at http://crd.lbl.gov/ dhbailey/dhbpapers/QRS.pdf.

[4] David H. Bailey, Yozo Hida, Xiaoye S. Li and Brandon Thompson, "ARPREC: An Arbitrary Precision Computation Package," Sept 2002, available at http://crd.lbl.gov/ dhbailey/dhbpapers/arprec.pdf.

[5] David H. Bailey, Xiaoye S. Li and K. Jeyabalan, "A comparison of three high-precision quadrature schemes," Experimental Mathematics, vol. 14 (2005), 317-329.

[6] D. Borwein and J. M. Borwein, "Some remarkable properties of sinc and related integrals," Ramanujan Journal, vol. 5 (2001), 73-90.

[7] Robert Baillie, David Borwein and Jonathan Borwein, "Some sinc sums and integrals," American Mathematical Monthly, 115 (10) (2008), 888-901. [D-drive Preprint 332].

[8] D. Borwein, J. M. Borwein and I. E. Leonard, " $L_{p}$ Norms and the Sinc Function," American Mathematical Monthly, in press, June 2009, available at http://users.cs.dal.ca/ jborwein/sincnorm.pdf.

[9] D. Borwein, J. M. Borwein, and B. Mares, "Multi-variable sinc integrals and volumes of polyhedra," Ramanujan Journal, vol. 6 (2002), 189-208.

[10] Peter Borwein, "An Efficient Algorithm for the Riemann Zeta Function," 1995, available at http://www.cecm.sfu.ca/preprints/1995pp.html.

[11] T. J. Bromwich, Theory of Infinite Series, First Edition 1908, Second Edition 1926, Blackie \& Sons, Glasgow.

[12] H. S. Carslaw, An Introduction to the Theory of Fourier's Series and Integrals, Third Revised Edition, Dover Publications Inc., New Jersey, 1950.

[13] D. J. Connon, "Fourier series representations of the Euler gamma function and the Barnes multiplegamma functions," preprint, Mar 2009, available at http://arxiv.org/ftp/arxiv/papers/0903/0903.4323.pdf.

[14] N. G. de Bruijn, Asymptotic Methods in Analysis, Second Edition, North-Holland Publishing Co., Amsterdam, 1961.

[15] A. Erdlyi et al, Higher transcendental functions. Vols. 1-3. Based on notes left by Harry Bateman. McGraw-Hill, New York, 1953-1955.

[16] W. B. Gearhart and H. S. Schultz, "The function $\frac{\sin (x)}{x}$," The College Mathematics Journal, vol. 2 (1990), 90-99.

[17] P. Henrici, Applied and Computational Complex Analysis Volume 2, John Wiley \& Sons, Inc., New York, 1977.

[18] I. E. Leonard and James Duemmel, "More-and Moore-Power Series without Taylor's Theorem," The American Mathematical Monthly, vol. 92 (1985), 588-589.

[19] F. W. J. Olver, Asymptotics and Special Functions (AKP Classics), Second Edition, AK Peters, Nattick, Mass, 1997.

[20] T. Ooura and M. Mori, "Double exponential formulas for oscillatory functions over the half infinite interval," Journal of Computational and Applied Mathematics, vol. 38 (1991), 353-360.

[21] F. Stenger, Numerical Methods Based on Sinc and Analytic Functions, Springer Series in Computational Mathematics, vol. 20, Springer-Verlag, New York, 1993.

[22] K. R. Stromberg, An Introduction to Classical Real Analysis, Wadsworth, Belmont, CA, 1981.

[23] H. Takahasi and M. Mori, "Double exponential formulas for numerical integration," Publications of RIMS, Kyoto University, vol. 9 (1974), 721-741. 